\title{
In vitro expression of Streptococcus pneumoniae ply gene in human monocytes and pneumocytes
}

\author{
Da-Kang Hu${ }^{1}$, Yang Liu², Xiang-Yang $\mathrm{Li}^{3^{*}}$ and Ying $\mathrm{Qu}^{1^{*}}$
}

\begin{abstract}
Background: Streptococcus pneumoniae is one major cause of pneumonia in human and contains various virulence factors that contribute to pathogenesis of pneumococcal disease. This study investigated the role of pneumolysin, Ply, in facilitating S. pneumoniae invasion into the host blood stream.

Methods: S. pneumoniae strains were isolated from clinical blood and sputum samples and confirmed by PCR. Expression of ply gene was assessed by infecting human monocytes and pneumocytes.

Results: A total of 23 strains of S. pneumoniae isolated from blood $(n=11)$ and sputum $(n=12)$ along with S. pneumoniae ATCC49619 were used to infect human monocyte (THP-1) and Type Il pneumocyte (A549) cell lines. All clinical strains of S. pneumoniae showed higher expression of ply mRNA than the American Type Culture Collection (ATCC) strain. Among the clinical strains, blood isolates showed higher expression of ply genes than sputum isolates, i.e., $2^{1.5}$ - to $2^{1.6}$-folds in THP-1 and $2^{0.4}$ - to $2^{4.9}$-folds in A549 cell lines.

Conclusions: The data from the current study demonstrated that ply gene of blood- and sputum-derived S. pneumoniae is differentially expressed in two different cell lines. Under survival pressure, ply is highly expressed in these two cell lines for blood-derived S. pneumoniae, indicating that ply gene may facilitate S. pneumoniae invasion into the host blood system.
\end{abstract}

Keywords: Streptococcus pneumoniae, Infection, Virulence gene, Pneumolysin (ply)

\section{Background}

Streptococcus pneumoniae (S. pneumoniae) is a grampositive diplococcus that is the main pathogenic bacterium of community-acquired pneumonia, otitis media, meningitis, abscesses, and other infections, particularly in infants and the elderly [1]. In developing countries, up to 1 million deaths annually are caused by pneumonia in children less than 5 years of age, in which S. pneumoniae is the major pathogenic bacterium [2]. Normally, $S$. pneumoniae is colonized in the respiratory tract of asymptomatic carriers as an opportunistic bacterium [3]. And it is difficult to predict when it converts to pathogenic bacterium although Manso et al. [4] reported that such a phase variation consists of genetic rearrangements.

\footnotetext{
* Correspondence: 89434436@qq.com; $1011575993 @ q q . c o m$

${ }^{3}$ Department of Laboratory Medicine, The Second Affiliated Hospital of Wenzhou Medical University, 109\# College West Road, Wenzhou 325027, China

'Department of Laboratory Medicine, Taizhou Municipal Hospital, 381\# Zhongshan East Road, Taizhou 318000, China

Full list of author information is available at the end of the article
}

However, when the host immunosystem becomes weak, S. pneumoniae will be infectious. During S. pneumoniae conversion from an opportunistic bacterium to a particularly pathogenic bacterium that causes severe invasive infections such as blood and cerebrospinal fluid (CSF) infections, the complex interaction will occur between $S$. pneumoniae and the body's immune system. In addition, infection could also be due to acquisition of virulent serotypes not previously part of the colonizing serotypes, which is named as transformation. Multiple components of S. pneumoniae, such as capsule and other virulence factors are involved in triggering the host immune responses [5-7], while a variety of cells in the host immune system (such as neutrophils, monocytes/macrophages, and dendritic cells) will be activated to defend against the infection by either killing the bacteria or releasing a variety of factors (such as IL-1 $\alpha$, IL-1 $\beta$, IL-6, IFN- $\alpha$, IL-8, and ICAM-1).

The septicemia and meningitis caused by S. pneumoniae are much more fatal than that of other types of 
infections [8]. However, few studies have been reported that focus on the mechanism responsible for S. pneumoniae invasion into the host blood system and nervous system [7]. Mahdi et al. [9] confirmed the role of transcription factor SP_0927 in pathogenesis and virulence. Uchiyama et al. [7] reported that neuraminidase A (NanA) is a virulence factor that facilitates S. pneumoniae invasion into the CSF. Ricci et al. [10] reported that pneumococcal surface protein A (PspA) contributes to pneumococcal meningitis rather than pneumococcal surface protein C (PspC). Based on previous studies on $S$. pneumoniae infection, different virulence factors in $S$. pneumoniae are able to induce multiple reactions from the host immune system. To date, there are more than 10 virulence factors discovered in S. pneumoniae, including NanA, capsular polysaccharide synthesis A (CpsA), choline-binding protein A (CbpA), pneumococcal surface adhesion A (PsaA), PspA, PspC, pneumolysin (Ply), and so on [2]. Different virulence factors may have different functions in contributing to the different types of S. pneumoniae infections.

Furthermore, study on S. pneumoniae virulence factors uses human cell lines, such as monocytes or lung epithelial cells. These types of cells are important in our body to prevent or defend against S. pneumoniae infection. The major role of monocytes is to kill bacteria and to trigger leukocyte chemotaxis for immunoreactions, while lung epithelial cells may function as an antigen presenter. In addition, type II pneumocytes have long been known to synthesize and secrete complement component C3, providing a target for S. pneumoniae adherence to those cells via CbpA. Thus, both types of cells are the most commonly used for study of the pathogenic mechanism of S. pneumoniae in vitro. To date, there is no report showing which specific virulence factor facilitates S. pneumoniae invasion into the host blood system. Possibly, different virulence factors play different roles in disease progression. In this paper, only ply expression is shown. We first isolated S. pneumoniae (23 strains) from clinical blood and sputum samples. They then infected monocytes or lung epithelial cell lines and the levels of ply mRNA expression were analyzed.

\section{Methods}

The experimental protocol was established according to the ethical guidelines of the Helsinki Declaration and was approved by the Human Ethics Committee of Taizhou Municipal Hospital, China. Written informed consent was obtained from individual participants.

\section{Isolation of S. pneumoniae strains}

The standard S. pneumoniae strain ATCC49619 was provided by the Chinese National Center for Medical Culture Collections (Beijing, China). Twenty-three clinical
S. pneumoniae strains were isolated from different inpatients at the Second Affiliated Hospital of Wenzhou Medical University (Wenzhou, Zhejiang, China) in 2009, which were preserved in $20 \%$ glycerin Luria-Bertani Medium and stored at $-80^{\circ} \mathrm{C}$; among them, 11 strains were isolated from blood samples, called blood-derived S. pneumoniae, and the other 12 strains were isolated from sputum samples, called sputum-derived $S$. pneumoniae. All 23 strains had no duplication and only one strain was isolated from each selected patient. All S. pneumoniae strains were identified by the Gram-Positive Identification Card (GPI) of VITEK-32 automatic microorganism analyzer (bioMérieux Co., Marcy-Etoile, France) and confirmed by PCR according to du Plessis [11].

\section{S. pneumoniae culture}

A total of 24 S. pneumoniae strains were cultured on blood agar plates at $37^{\circ} \mathrm{C}$ in a $5 \% \mathrm{CO}_{2}$ incubator (Thermo Electron Co., Waltham, MA, USA) and adjusted to 1.0 McFarland in normal saline, which is used for infection experiments and RNA extraction.

\section{Cell line and culture}

Human monocytes THP-1 and type II pneumocytes A549 cell lines were obtained from the Cell Bank of Chinese Academy of Sciences (Shanghai, China) and cultured with RPMI 1640 and F-12 K medium (GIBCO, CA, USA), respectively, and $10 \%$ fetal bovine serum (Zhejiang Tianhang Biological Technology Co., Zhejiang, China) plus 1\% strep-penicillin (Beijing Solarbio Science \& Technology Co., Beijing, China) in a $37^{\circ} \mathrm{C}$ humidified incubator (Thermo Electron Co., Waltham, MA, USA) with 95\% air and 5\% $\mathrm{CO}_{2}$. THP-1 and A549 cells were prepared at concentrations of 4.0 and $3.0 \times 10^{8} / \mathrm{L}$ respectively for the infection experiments and such preparation was conducted between May 2011 and July 2011 at the Center Laboratory of Experiments, Wenzhou Medical University.

\section{Infection of THP-1 and A549 cell lines with S. pneumoniae cultures}

One milliliter of THP-1 cells was grown in 24-well culture plates, which were $4.0 \times 10^{8} / \mathrm{L}$. One milliliter of $S$. pneumoniae cultures from clinical samples and American Type Culture Collection (ATCC) strains was then seeded into the 24-well culture plates, which was 1.0 McFarland. The blank control used normal saline instead of S. pneumoniae. The cells were maintained at $37^{\circ} \mathrm{C}$ in $5 \% \mathrm{CO}_{2}$ for $4 \mathrm{~h}$ (25 wells) and $8 \mathrm{~h}$ (other 25 wells). At the end of the experiments, all of the cell cultures plus controls were transferred into $1.5-\mathrm{ml}$ centrifuge tubes and centrifuged for $10 \mathrm{~min}$ at $4,000 \mathrm{rpm}$ and then subjected to RNA isolation and PCR analysis. For A549 cell cultures, $50 \mu \mathrm{l}$ of $0.25 \%$ trypsin (GIBCO, Pleasanton, CA, USA) were used to digest the adherent 
cells after the suspensions were harvested, whereas there was no trypsin used for THP-1 cell suspension culture. The experiments were repeated at least once and all the 24 S. pneumoniae strains were studied in these two cell lines.

\section{Semi-quantitative RT-PCR}

Total RNA from S. pneumoniae culture was isolated using a RNA extraction kit from Takara (Dalian, China) according to the manufacturer's protocols. The purity of RNA was determined by the Nanodrop 2000 spectrophotometer (Thermo Electron Co., Waltham, MA, USA) and the optical density ratios of OD260/OD280 were all between 1.8 and 2.2. After that, these RNA samples were converted to cDNA using $2.0 \mu \mathrm{l}$ of $5 \mathrm{x}$ PrimeScript Buffer, $0.5 \mu \mathrm{l}$ PrimeScript Buffer RT Enzyme Mix I, $0.5 \mu \mathrm{l}$ Oligo dT Primer $(50 \mu \mathrm{M}), 0.5 \mu \mathrm{l}$ Random 6 mers $(100 \mu \mathrm{M}), 5.0 \mu \mathrm{l}$ total RNA, and $1.5 \mu \mathrm{l}$ RNase free $\mathrm{ddH}_{2} \mathrm{O}$ for $15 \mathrm{~min}$ at $37^{\circ} \mathrm{C}$ and then stopped at $85^{\circ} \mathrm{C}$ for $5 \mathrm{~s}$. After that, real-time PCR analysis was performed by using $10.0 \mu \mathrm{l}$ SYBR Premix Ex Taq II (Takara), $0.8 \mu \mathrm{l}$ PCR primer each $(10 \mu \mathrm{M}), 0.4 \mu \mathrm{l}$ ROX Reference Dye II (50x), $2.0 \mu \mathrm{l}$ RT mixture, and $6.0 \mu \mathrm{l}$ $\mathrm{dd}_{2} \mathrm{O}$ for pre-degeneration at $95^{\circ} \mathrm{C}$ for $30 \mathrm{~s}$ and then 40 cycles of $95^{\circ} \mathrm{C}$ for $5 \mathrm{~s}$ and $60^{\circ} \mathrm{C}$ for $34 \mathrm{~s}$ in a 7,500 fluorescence quantitative PCR instrument (ABI, San Ramon, CA, USA). The ply primers were synthesized according to a previous study [12] (5'-GATGGCAAA TAAAGCAGTAAATGACT- $3^{\prime}$ and $5^{\prime}$-TGATGCCAC TTAGCCAACAAATCG-3'). The $16 \mathrm{~S}$ rRNA primers were synthesized according to reference [13]: (5'GGTGAGTAACGCGTAGGTAA-3' and 5'-ACGATC CGAAAACCTTCTTC-3'). All the primers were synthesized at Shinegene Co. (Shanghai Shanjing, China). Levels of $16 \mathrm{~S}$ rRNA were used as an internal reference to quantify the level of ply expression in this study. The blank control was from non-infected cell lines to replace $S$. pneumoniae with normal saline. PCR amplification data were quantified by using ABI 7500 Software v2.0.1. while 'Threshold' and 'Baseline' were selected as 'auto'.

\section{Statistical analysis}

SPSS17.0 statistical software (SPSS, Chicago, IL, USA) was used for all statistical analyses. The normality analysis was performed by using the Kolmogorov-Smirnov test, and the homogeneity of variance analysis between two groups using Levene's test. The mean difference between clinical S. pneumoniae (including blood and sputum S. pneumoniae samples) and control or ATCC49619 was analyzed using the single-sample $t$ test, and the comparison of blood- and sputum-derived S. pneumoniae samples using analysis of variance for factorial designs in infection of A549 cells. In addition, the two-sample $t$ test was used to determine whether there is a difference in virulence factor expressions between blood group and sputum group of $S$. pneumoniae before the infection. The Cochran \& Cox separate variance estimation $t$ test was used among blood or sputum groups of $S$. pneumoniae after the infection of THP-1 cells. $P>0.10$ in the normality test, $P>0.10$ in the variance homogeneity test, and $P<0.05$ in mean comparison were considered statistically significant.

\section{Results}

\section{Expression of virulence gene ply in S. pneumoniae after} infecting THP-1 and A549 cells

To detect the changes in expression of virulence gene ply in these S. pneumoniae samples, we used the S. pneumoniae samples to infect THP-1 and A549 cells for $4 \mathrm{~h}$ and $8 \mathrm{~h}$, respectively.

As shown in Table 1, $\Delta \mathrm{Ct}$ values of the virulence gene ply were significantly different after blood-derived S. pneumoniae infected THP-1 cells for $4 \mathrm{~h}$ and $8 \mathrm{~h}$ compared to pre-infection, but there was no significant difference between $4 \mathrm{~h}$ and $8 \mathrm{~h}$ infection. However, there was no statistically significant difference in expression of ply after sputum-derived S. pneumoniae infected THP-1 cells for $4 \mathrm{~h}$ and $8 \mathrm{~h}$ compared to pre-infection, but the expression was significantly higher after $8 \mathrm{~h}$ infection. However, compared to the ATCC49619 control, before and after blood- or sputum-derived $S$. pneumoniae infected THP-1 cells, $\Delta$ Ct values of virulence gene ply had a significant difference, indicating that the expression of ply was weaker than ATCC49619 before infection, but expression of ply was higher than ATCC49619 after infection. Between blood- and sputum-derived S. pneumoniae, $\Delta \mathrm{Ct}$ of ply expression were significantly different after they infected THP-1 cells for 8 h: after infection in THP-1 cells for $8 \mathrm{~h}$, ply expression of blood-derived S. pneumoniae was stronger than that of sputum-derived ones. Similar results of ply expression were obtained from blood-derived or sputum-derived S. pneumoniae infecting A549 cells for $4 \mathrm{~h}$ and $8 \mathrm{~h}$ (Table 1$)$.

In A549 cells, there was no statistically significant difference in expression of virulence gene ply between the following groups: the blood group vs. the ATCC49619 standard in $4 \mathrm{~h}$ or $8 \mathrm{~h}$ cultures, the sputum group vs. the ATCC49619 standard in 4 h cultures $(t=-0.901,-1.049,-1.206$, respectively; $P=0.389$, $0.319,0.253$, respectively). In contrast, the remaining comparisons showed significant differences $(P<0.05)$ (Tables 1 and 2). Moreover, after both blood- and sputumderived S. pneumoniae infected A549 cells, there were statistically significant differences in expression of virulence gene ply between blood- and sputum-derived S. pneumoniae $(F=6.560, P=0.014)$, but $4 \mathrm{~h}$ to $8 \mathrm{~h}$ culture did not 
Table 1 Expression of virulence gene ply after S. pneumoniae infected THP-1 or A549 cells

\begin{tabular}{|c|c|c|c|c|c|c|}
\hline \multirow[t]{2}{*}{ Groups } & \multirow[t]{2}{*}{$n$} & \multicolumn{5}{|c|}{ ply $\left(\Delta \mathrm{Ct}^{-} \mathrm{X} \pm s\right)$} \\
\hline & & $\mathrm{Oh}$ & $4 \mathrm{~h}$ (THP-1) & $8 \mathrm{~h}(\mathrm{THP}-1)$ & 4 h (A549) & 8 h (A549) \\
\hline ATCC & 1 & 16.2 & 31.5 & 27.9 & 21.8 & 19.1 \\
\hline Blood SP & 11 & $29.2 \pm 2.6$ & $24.8 \pm 3.2$ & $23.0 \pm 1.5$ & $20.4 \pm 5.1$ & $18.2 \pm 2.9$ \\
\hline Sputum SP & 12 & $28.1 \pm 2.9$ & $26.3 \pm 2.7$ & $24.6 \pm 1.4$ & $20.8 \pm 3.0$ & $23.3 \pm 3.1$ \\
\hline
\end{tabular}

Note: $\Delta \mathrm{Ct}=$ Mean $\mathrm{Ct}$ of tested gene - Mean Ct of control gene. Smaller $\Delta \mathrm{Ct}$ means more ply expression. Level of 16S rRNA was used as a control, which expresses much higher than that of ply. SP, Streptococcus pneumoniae; ATCC, American Type Culture Collection.

show a significant difference $(F=0.025, P=0.874)$, analyzed by a variance of factorial designs. However, interaction between samples (including blood and sputum samples) and infection time points had statistical significance $(F=5.061, P=0.030)$.

Table 2 showed $P$ values between the following compared groups after S. pneumoniae infected THP-1 cells due to non-homogeneity of variance. Cochran \& Cox separate variance estimation $t$ test was used to compare blood and sputum groups of $S$. pneumoniae after the infection.

\section{Changes in THP-1 and A549 cell morphology after S. pneumoniae infection}

As shown in Figure 1, the longer time of THP-1 cells culture with $S$. pneumoniae, the more deaths THP-1 cells suffered, levels of which were associated with different sources of S. pneumoniae isolations and amounts. Similar data were observed in A549 cell cultures of these S. pneumoniae samples.

Table $2 \boldsymbol{P}$ values between the following compared groups after $S$. pneumoniae infected THP-1 cells

\begin{tabular}{lll}
\hline Compared groups & $\boldsymbol{t}$ & $\boldsymbol{P}$ value \\
\hline 0 h B-SP $(n=11)$ vs. 0 h S-SP $(n=12)$ & 0.956 & 0.350 \\
4 h B-SP $(n=11)$ vs. 4 h S-SP $(n=12)$ & -1.223 & 0.235 \\
8 h B-SP $(n=11)$ vs. 8 h S-SP $(n=12)$ & -2.690 & 0.014 \\
0 h B-SP $(n=11)$ vs. 4 h B-SP $(n=11)$ & 2.778 & 0.020 \\
0 h B-SP $(n=11)$ vs. 8 h B-SP $(n=11)$ & 6.775 & $<0.001$ \\
4 h B-SP $(n=11)$ vs. 8 h B-SP $(n=11)$ & 1.578 & $>0.10$ \\
0 h S-SP $(n=12)$ vs. 4 h S-SP $(n=12)$ & 1.561 & 0.147 \\
0 h S-SP $(n=12)$ vs. 8 h S-SP $(n=12)$ & 4.341 & 0.001 \\
4 h S-SP $(n=12)$ vs. 8 h S-SP $(n=12)$ & 2.034 & $>0.05$ \\
0 h B-SP $(n=11)$ vs. 0 h ATCC $(n=1)$ & 16.449 & $<0.001$ \\
4 h B-SP $(n=11)$ vs. 4 h ATCC $(n=1)$ & -6.908 & $<0.001$ \\
8 h B-SP $(n=11)$ vs. 8 h ATCC $(n=1)$ & -11.154 & $<0.001$ \\
0 h S-SP $(n=12)$ vs. 0 h ATCC $(n=1)$ & 14.350 & $<0.001$ \\
4 h S-SP $(n=12)$ vs. 4 h ATCC $(n=1)$ & -6.706 & $<0.001$ \\
8 h S-SP $(n=12)$ vs. 8 h ATCC $(n=1)$ & -7.859 & $<0.001$ \\
\hline
\end{tabular}

Note: SP, Streptococcus pneumoniae; B-SP, blood SP; S-SP, sputum SP; ATCC, American Type Culture Collection.

\section{Discussion}

In the current study, we first isolated and confirmed 23 S. pneumoniae strains from clinical blood and sputum samples. We then used these 23 S. pneumoniae strains and ATCC49619 to infect human monocytes and pneumocytes and then analyzed expression of the S. pneumoniae virulence gene ply in these 23 strains and ATCC49619. We found that levels of ply mRNA expression were much higher in clinical $S$. pneumoniae samples than in the ATCC49619 standard and in bloodderived S. pneumoniae samples than in sputum-derived ones. This finding demonstrated differential expression of ply mRNA levels in blood-derived S. pneumoniae after infecting THP-1 and A549 cells compared to sputumderived ones. This study provides indirect molecular evidence that the $S$. pneumoniae virulence gene ply may facilitate $S$. pneumoniae invasion into the host blood system.

Indeed, Ply is a pore-forming toxin with a molecular weight of $53 \mathrm{kD}$ and is known as a cholesterol-binding cytolysin, a key virulence factor for clinical S. pneumoniae strains. Ply protein can dissolve cell membranes in almost all eukaryotes. Specifically, Ply toxin will bind to a cholesterol-rich membrane using its pore-forming mechanism and then penetrate into the lipid bilayer of the cell membrane and oligomerize to form a perforation on the cell membrane [14]. Ply can also activate macrophages to execute apoptosis without membrane pore formation [15]. Thus, Ply toxin has a wide range of biological activities, such as facilitation of $S$. pneumoniae colonization and pathogenicity. Ply can also activate the classic complement pathway in the human body, inhibit cough and bactericidal activity and migration of white blood cells [16], stimulate IL-8 synthesis [17], and increase ICAM-1 and IL-1 $\beta$ secretions [18-20]. The Ply impact on the immune response to the pneumococcus is highly dependent on the strain background, thus, it is surely important of the interaction between specific virulence factors and other components of the genetic background of this organism [19]. A recent study also confirmed the role of ply in biofilm formation, which is separate from the hemolytic activity responsible for tissue damage during pneumococcal disease [21]. Our current data showed that after infecting THP-1 cells, 


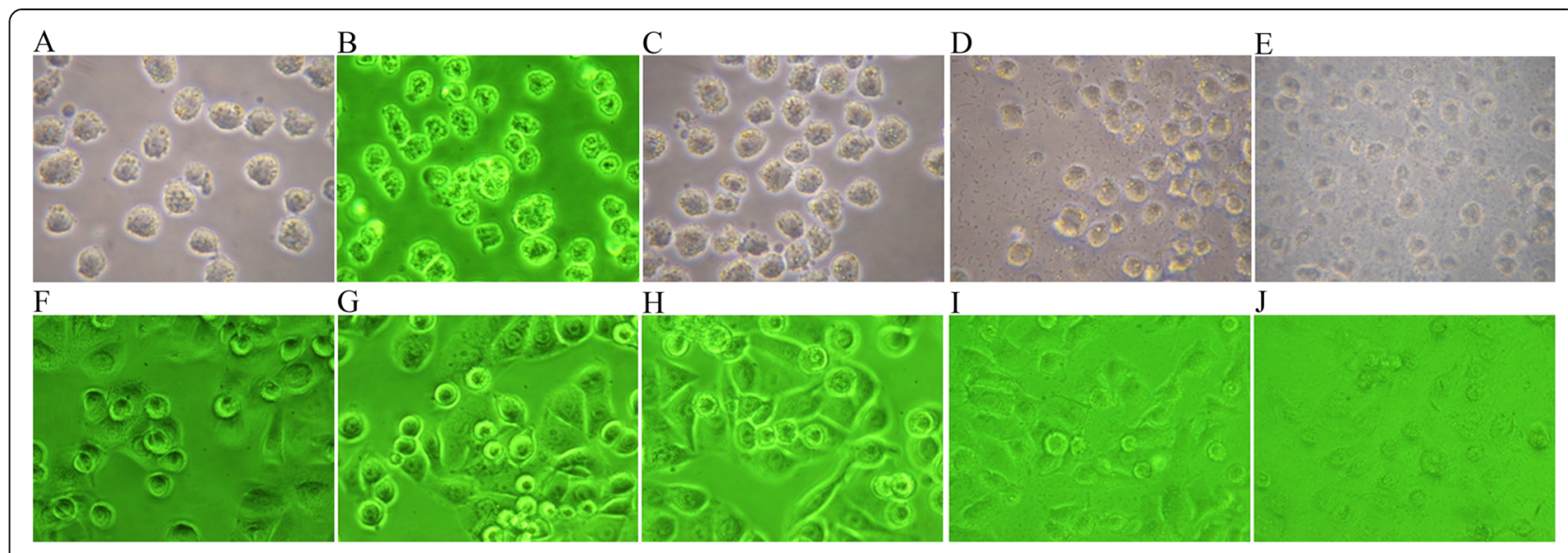

Figure 1 Morphological changes of THP-1 and A549 cells after Streptococcus pneumoniae (SP) infection. THP-1 and A549 cells began to die after infection at 4 to 8 h: (A) THP-1 at 0 h; (B) THP-1 (uninfected control) at 4 h; (C) THP-1 (uninfected control) at 8 h; (D) THP-1 with SP at 4 h; (E) THP-1 with SP at 8 h; (F) A549 at 0 h; (G) A549 (uninfected control) at 4 h; (H) A549 (uninfected control) at 8 h; (I) A549 with SP at 4 h; (J) A549 with SP at $8 \mathrm{~h}$. (A-J: $\times 400)$. One strain of blood-derived SP was used except in the uninfected control. $0 \mathrm{~h}$ means before infection.

expression of ply mRNA was significantly induced in blood-derived $S$. pneumoniae strains compared to sputum-derived ones, indicating that the increased ply expression may contribute to $S$. pneumoniae against defense of THP-1 cells, which may be one of the mechanisms for S. pneumoniae invasion into the host blood system. In the current study, we determined ply expression after infecting two types of human cell lines and data are identical, which are consistent with a previous study [22]. Besides, as a whole, expression of psaA and cpsA was also studied in a previous study [23]. And different patterns of their expression clearly showed different roles of such three virulence genes. The expression of cpsA is the basis of the pathogenicity of SP and ply expression is more important than that of psaA in SP invasion into the blood system. Our current data demonstrated that inflammation induction of different SP sources tends to be consistent [20]. However, the infection types are determined mainly by different sources of SP itself, especially its expression of virulence genes.

In the current study, we utilized a standard strain of S. pneumoniae ATCC49619 for comparison. We found that S. pneumoniae isolated from both blood and sputum samples were more virulent by expression of high levels of Ply toxin after infecting THP-1 and A549 cells. ATCC49619 is a standard S. pneumoniae strain and its physical and chemical properties are relatively stable. The S. pneumoniae isolated from clinical samples were much more easily influenced by external environment than ATCC49619. Thus, compared to clinical S. pneumoniae, the levels of virulence and viability of ATCC49619 are weaker, all of which may indicate that clinical S. pneumoniae strains are more infectious.
However, it remains to be determined whether S. pneumoniae isolated from different individuals also have different levels of infection potential.

Moreover, we also showed the interaction between S. pneumoniae and THP-1 cells or A549 cells. Different sources of S. pneumoniae resulted in different outcomes, which could suggest that various strains of $S$. pneumoniae have different abilities to infect the lung, blood, or even the CSF. However, this study is just a proof-ofprinciple and much more are needed to investigate other virulence factors of S. pneumoniae or their combinations in infecting the human body. Indeed, S. pneumoniae have many other virulence genes, such as nanA [7], which has been demonstrated to facilitate $S$. pneumoniae invasion into the CSF. Moreover, S. pneumoniae invasion into the host blood system is an important step and prerequisite for its further invasion into the CSF in most cases. The role of Ply toxin in S. pneumoniae invasion into the host blood system and its relationship with NanA are worthy of further investigation. In addition, the significance of this in vitro study needs further confirmation by an in vivo study.

\section{Conclusions}

The current study demonstrated that ply gene of bloodand sputum-derived S. pneumoniae is differentially expressed in two different cell lines. Under survival pressure, higher expression of ply gene is needed in these two cell lines for blood-derived S. pneumoniae, which indicates that ply gene may facilitate S. pneumoniae invasion into the host blood system.

\section{Competing interests}

The authors declare that they have no competing interests. 


\section{Authors' contributions}

DKH and XYL defined the research theme. YL designed methods and experiments and carried out the laboratory experiments. YQ analyzed the data and wrote the paper. All authors read and approved the final manuscript.

\section{Acknowledgements}

This work was supported in part by a grant from The Science and Technology Development Planning (\#10275) funds of Jiaojiang District, Taizhou City, China.

\section{Author details}

'Department of Laboratory Medicine, Taizhou Municipal Hospital, 381\# Zhongshan East Road, Taizhou 318000, China. ${ }^{2}$ Department of Laboratory Medicine, The First Affiliated Hospital of Nanchang University, 17\# Yong Wai Zheng Street, Nanchang 330006, China. ${ }^{3}$ Department of Laboratory Medicine, The Second Affiliated Hospital of Wenzhou Medical University, 109\# College West Road, Wenzhou 325027, China.

Received: 5 December 2014 Accepted: 27 April 2015

Published online: 07 May 2015

\section{References}

1. Jin P, Xiao M, Kong F, Oftadeh S, Zhou F, Liu C, et al. Simple, accurate, serotype-specific PCR assay to differentiate Streptococcus pneumoniae serotypes 6A, 6B, and 6C. J Clin Microbiol. 2009:47:2470-4.

2. Mitchell AM, Mitchell TJ. Streptococcus pneumoniae: virulence factors and variation. Clin Microbiol Infect. 2010;16:411-8.

3. Suzuki H, Ikeda K. Mode of action of long-term low-dose macrolide therapy for chronic sinusitis in the light of neutrophil recruitment. Curr Drug Targets Inflamm Allergy. 2002;1:117-26.

4. Manso AS, Chai MH, Atack JM, Furi L, De Ste Croix M, Haigh R, et al. A random six-phase switch regulates pneumococcal virulence via global epigenetic changes. Nat Commun. 2014;5:5055.

5. Cockeran R, Mitchell TJ, Feldman C, Anderson R. Pneumolysin induces release of matrix metalloproteinase- 8 and -9 from human neutrophils. Eur Respir J. 2009:34:1167-70.

6. Morona JK, Morona R, Paton JC. Attachment of capsular polysaccharide to the cell wall of Streptococcus pneumoniae type 2 is required for invasive disease. Proc Natl Acad Sci U S A. 2006;103:8505-10.

7. Uchiyama S, Carlin AF, Khosravi A, Weiman S, Banerjee A, Quach D, et al. The surface-anchored NanA protein promotes pneumococcal brain endothelial cell invasion. J Exp Med. 2009;206:1845-52.

8. Weisfelt M, van de Beek D, Spanjaard L, Reitsma JB, de Gans J. Clinical features, complications, and outcome in adults with pneumococcal meningitis: a prospective case series. Lancet Neurol. 2006;5:123-9.

9. Mahdi LK, Ebrahimie E, Adelson DL, Paton JC, Ogunniyi AD. A transcription factor contributes to pathogenesis and virulence in Streptococcus pneumoniae. PLoS One. 2013;8, e70862.

10. Ricci S, Gerlini A, Pammolli A, Chiavolini D, Braione V, Tripodi SA, et al. Contribution of different pneumococcal virulence factors to experimental meningitis in mice. BMC Infect Dis. 2013;13:444.

11. du Plessis M, Smith AM, Klugman KP. Rapid detection of penicillin-resistant Streptococcus pneumoniae in cerebrospinal fluid by a seminested-PCR strategy. J Clin Microbiol. 1998;36:453-7.

12. Walker JA, Allen RL, Falmagne P, Johnson MK, Boulnois GJ. Molecular cloning, characterization, and complete nucleotide sequence of the gene for pneumolysin, the sulfhydryl-activated toxin of Streptococcus pneumoniae. Infect Immun. 1987:55:1184-9.

13. Bentley RW, Leigh JA, Collins MD. Intrageneric structure of Streptococcus based on comparative analysis of small-subunit rRNA sequences. Int J Syst Bacteriol. 1991:41:487-94.

14. Palmer M. The family of thiol-activated, cholesterol-binding cytolysins. Toxicon. 2001;39:1681-9.

15. Bewley MA, Naughton M, Preston J, Mitchell A, Holmes A, Marriott HM, et al. Pneumolysin activates macrophage lysosomal membrane permeabilization and executes apoptosis by distinct mechanisms without membrane pore formation. mBio. 2014:5:e01710-4.

16. Paton JC, Ferrante A. Inhibition of human polymorphonuclear leukocyte respiratory burst, bactericidal activity, and migration by pneumolysin. Infect Immun. 1983;41:1212-6.
17. Rogers PD, Thornton J, Barker KS, McDaniel DO, Sacks GS, Swiatlo E, et al Pneumolysin-dependent and -independent gene expression identified by CDNA microarray analysis of THP-1 human mononuclear cells stimulated by Streptococcus pneumoniae. Infect Immun. 2003;71:2087-94.

18. Thornton J, McDaniel LS. THP-1 monocytes up-regulate intercellular adhesion molecule 1 in response to pneumolysin from Streptococcus pneumoniae. Infect Immun. 2005;73:6493-8.

19. Harvey RM, Hughes CE, Paton AW, Trappetti C, Tweten RK, Paton JC. The impact of Pneumolysin on the macrophage response to Streptococcus pneumoniae is strain-dependent. PLoS One. 2014;9, e103625.

20. Zhang J, Hu DK, Wang DG, Liu Y, Liu CB, Yu LH, et al. Effects of clinical isolates of Streptococcus pneumoniae on THP-1 human monocytic cells. Mol Med Rep. 2013;8:1570-4

21. Shak JR, Ludewick HP, Howery KE, Sakai F, Yi H, Harvey RM. Novel role for the Streptococcus pneumoniae toxin pneumolysin in the assembly of biofilms. mBio. 2013;4:e00655-13.

22. Hirst RA, Yesilkaya H, Clitheroe E, Rutman A, Dufty N, Mitchell TJ, et al. Sensitivities of human monocytes and epithelial cells to pneumolysin are different. Infect Immun. 2002;70:1017-22.

23. Hu DK, Wang DG, Liu Y, Liu CB, Yu LH, Qu Y, et al. Roles of virulence genes (PsaA and CpsA) on the invasion of Streptococcus pneumoniae into blood system. Eur J Med Res. 2013;18:14

\section{Submit your next manuscript to BioMed Central and take full advantage of:}

- Convenient online submission

- Thorough peer review

- No space constraints or color figure charges

- Immediate publication on acceptance

- Inclusion in PubMed, CAS, Scopus and Google Scholar

- Research which is freely available for redistribution 